\title{
Meningo-Encephalomyelitis Due to the Saprophagous Nematode, Micronema Deletrix
}

\author{
JAN HOOGSTRATEN AND W. GERARD YOUNG*
}

SUMMARY: A five-year-old boy succumbed 24 days following an unusual farm accident in which considerable manure was deposited in multiple lacerations. Death was due to an extensive meningo-encephalomyelitis caused by a nematode that is ordinarily saprophagous.

RÉSUMÉ: Un garçon de cinq ans est mort 24 jours après un accident de ferme inhabituel dans lequel de l'engrais s'est logé dans plusieurs lacérations. La mort est due à une méningo-encéphalomyélite étendue causée par un nématode qui est ordinairement saprophage.

From The Department of Pediatric Pathology, Health Sciences Center, 685 Bannatyne Avenue, Winnipeg, Canada R3E OW1.

Reprint requests to Dr. J. Hoogstraten at The Health Sciences Center.

*Deceased April 3, 1974
Meningo-encephalomyelitis due to nematode infestation is an unusual event in humans. Its occurrence in northern temperate climes consequent to wound infestation, following trauma, must be extremely rare. The present report is the first known example of meningoencephalomyelitis in the human due to a saprophagous nematode which ordinarily dwells in decaying humus.

The subject of this report was a five-year-old boy who fell into and passed through the mechanism of a manure spreader, sustaining multiple facial lacerations, comminuted fracture of the mandible, a penetrating sucking chest wound, a penetrating abdominal wound with eventration of gut, and a large laceration of the buttock and thigh with fracture of the corresponding femur.

He received emergency therapy at his local village hospital. This consisted of a tracheostomy and closure of all wounds. He was then transferred 150 miles to the Children's Hospital of Winnipeg, arriving there eight hours after his accident. At no time did the child lose consciousness.

In Winnipeg, his wounds were re-opened, and considerable manure was removed. His leg was placed in traction. Six days later, his mandible was wired.

Eight days after his accident, his abdominal wound dehisced, with eventration of gut, again necessitating operation.

Twelve days following his acci- dent, secondary closure of his lacerations was performed. His condition was excellent at this time and it was thought that he would soon be returned to his local village hospital for convalescence.

Following this, however, his temperature began to rise each day to about 39.5 degrees. Pseudomonas aeruginosa was recovered from his wounds, but blood cultures remained sterile. He had been receiving carbenicillin intravenously in large doses, and gentamycin and penicillin were added to the therapeutic regimen.

Eighteen days following his injury, he began to exhibit lethargy. Lumbar puncture revealed fluid under normal pressure, but containing 95 cells per c.mm., predominantly lymphocytes and large macrophages. In the ensuing hours he began to develop fluctuating focal neurological signs, became more obtunded, and a repeat lumbar puncture revealed over 300 cells per c.mm., again approximately equal proportions of lymphocytes and large motile macrophages. A pneumoencephalogram suggested equivocal evidence of a space occupying lesion in the posterior fossa, but this was not confirmed by subsequent craniotomy. He succumbed 24 days following his accident with the clinical diagnosis of meningoencephalitis of unknown etiology.

Autopsy revealed no significant gross abnormalities except for what was interpreted as an unusual degree 


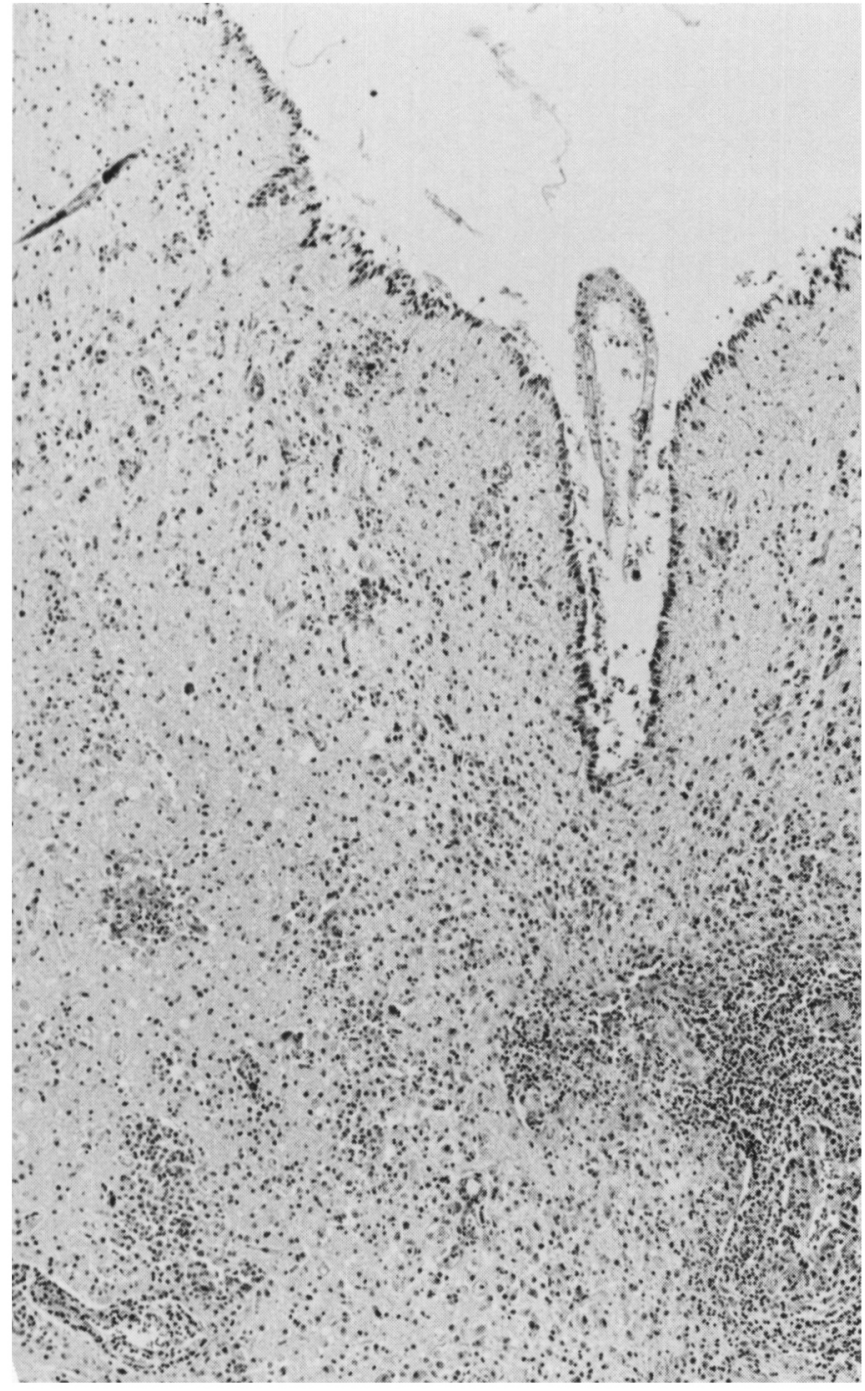

Figure 1-Heavy inflammatory infiltrate in floor of fourth ventricle. Nematodes in cross section are not evident at this magnification: A nematode in tangential longitudinal section is evident in the upper left. H. and E. x75.

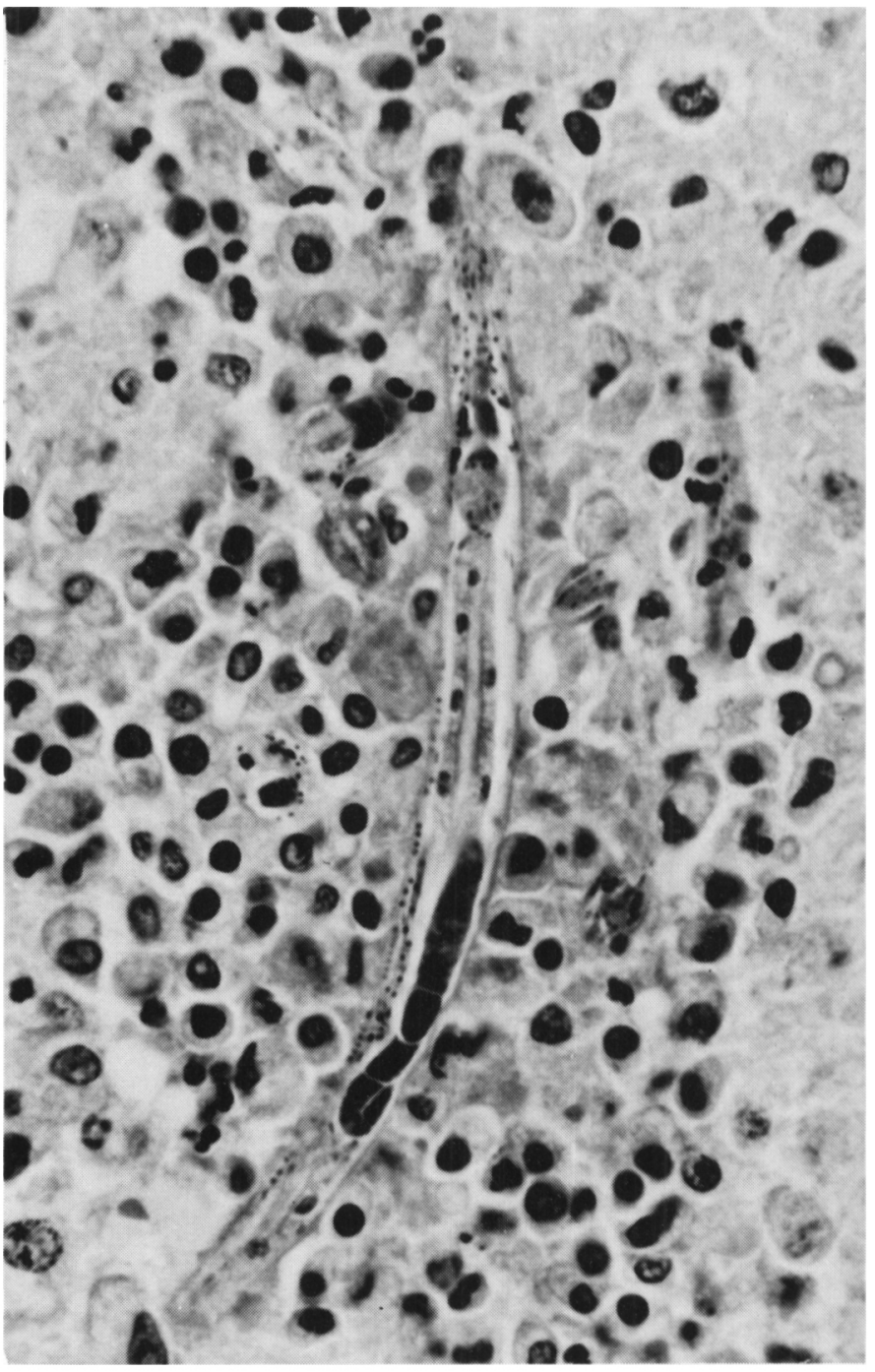

Figure 2-Nematode surrounded by inflammatory infiltrate, demonstrating the oesophageal bulb, intestine and darklystained gonad. The caudal end of the ovary is recurved dorsally. H. and E. x640. of hyperemia of the white matter. The leptomeninges appeared of normal thinness and transparency, with no gross evidence of inflammatory exudate. The intracranial venous sinuses were patent and normal. His multiple wounds appeared well healed and clean.

Microscopic examination, however, revealed an extensive and unusual meningo-encephalomyelitis.

Extensive inflammatory foci consisting of lymphocytes, swollen microglial cells or macrophages and rare multinucleated giant cells were scattered throughout the leptomeninges, grey and white matter of the cerebral hemispheres, pons, medulla, midbrain, cerebellum, and throughout the length of the spinal cord. Small numbers of neutrophile granulocytes were present in some areas. Eosinophiles were infrequent. None of the many sections examined were free of these inflammatory lesions. The inflammation was notably perivascular in distribution, but was not confined to that location (Figure 1).

Scattered within, and also outside of these inflammatory foci were nematodes in various stages of de- velopment (Figures 2, 3 and 4). Some were lying free within the central canal of the spinal cord. Some were penetrating the ependyma, lying partly within ventricular walls and partly within the lumen of the ventricles. Fibrin thrombi were sometimes evident within capillaries, and small perivascular hemorrhages were not infrequent. Eosinophilic remnants of effete nematodes were also evident within inflammatory foci within the choroid plexus.

A few inflammatory foci and organisms were present within the 


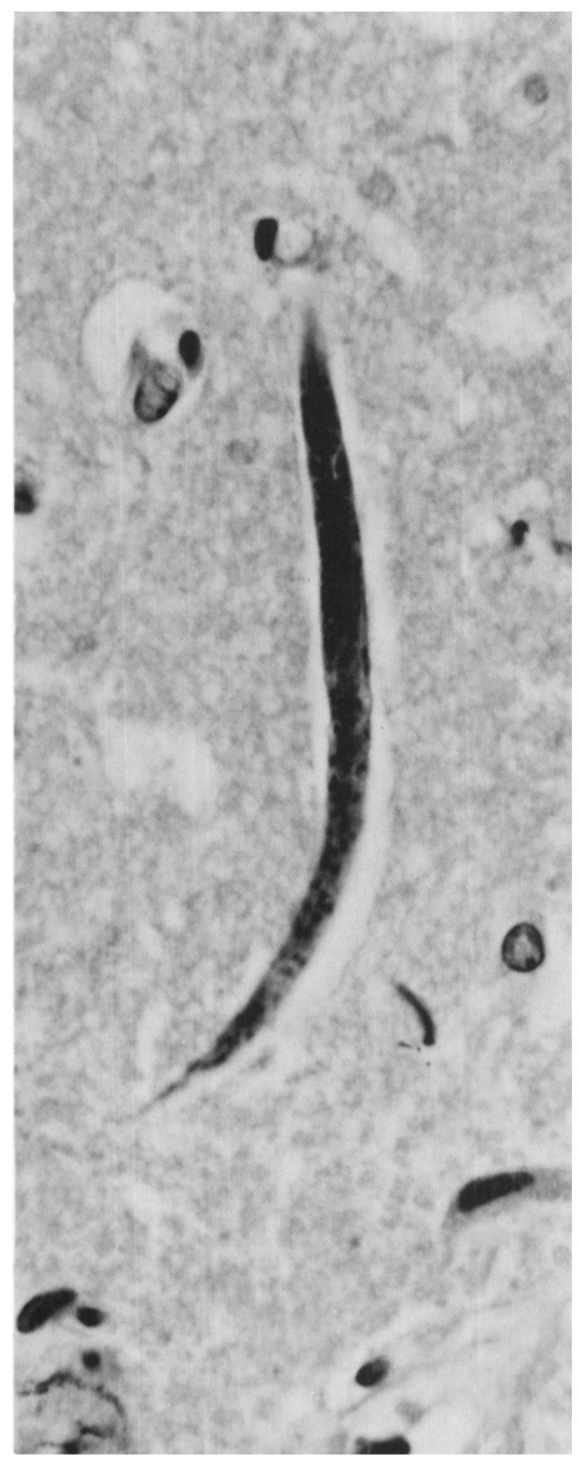

Figure 3-Juvenile or incompletely developed nematode. H. and E. x 640 .

posterior pituitary, and small numbers of juvenile or immature organisms were evident within the lungs, lying within capillaries in the alveolar walls (Figure 5).

No nematodes were evident in any other viscus, but there was a minimal, focal, myocardial, lymphocytic infiltrate. There was no involvement of the liver, and no nematodes were evident in the intestinal lumen or walls.

Although there were enormous numbers of organisms within this child's central nervous system, the plane of sections rarely included the whole length of a nematode. Moreover, these eel-worms were present in various stages of development. Estimates of the size of the most mature forms were therefore only approximate - suggesting a length of approximately $250 \mathrm{mic}$ rons, with a greatest diameter of approximately 15 microns.

These cylindrical organisms tapered gently anteriorly to a diameter of approximately 3.5 microns at the stoma, while the tail was more sharply tapered. The anterior parts of a mature organism are illustrated in Figure 6. The pharynx or stoma

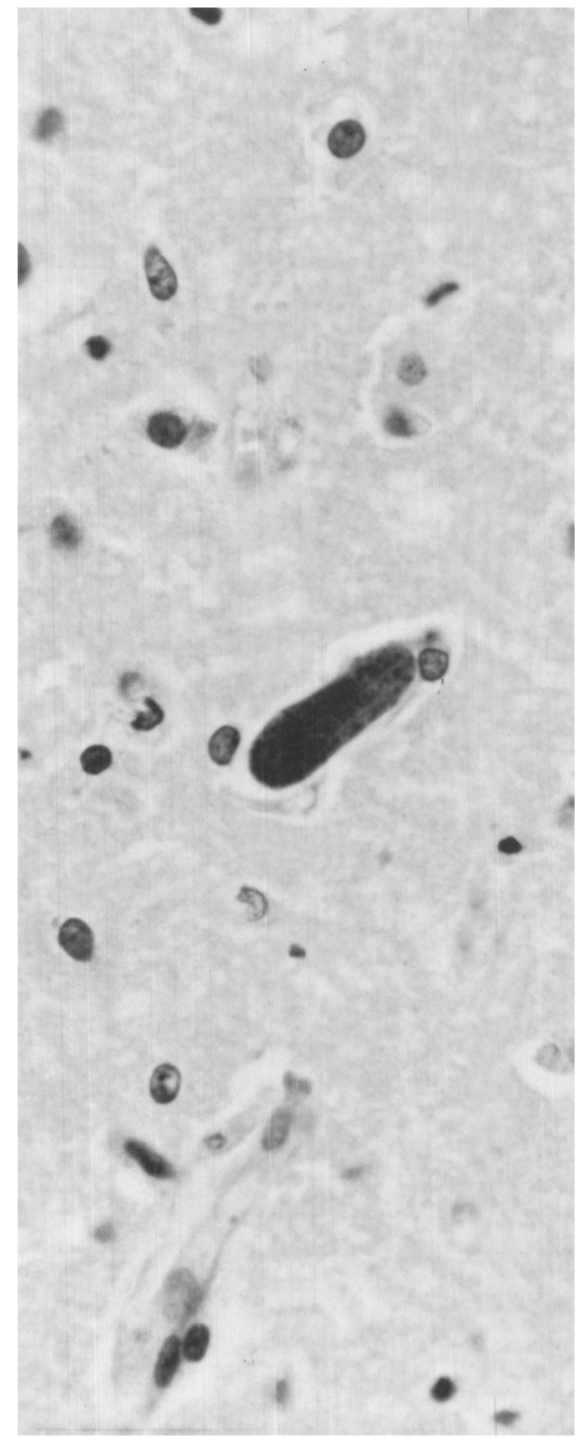

Figure 4-Small recurved or folded embryo. H. and E. x640.

measures approximately eight microns in length and the esophagus approximately 80 microns. The anterior 36 microns of this latter structure is a delicately elongated flask shape, and is connected to a posterior oval or pear-shaped bulb by a narrow isthmus approximately 27 microns in length.

Sections of the spinal cord were submitted to the United States Armed Forces Institute of Pathology. Studies performed by Dr. Daniel $\mathrm{H}$. Connor and his colleagues suggested that the anatomy of the anterior parts of this nematode was 


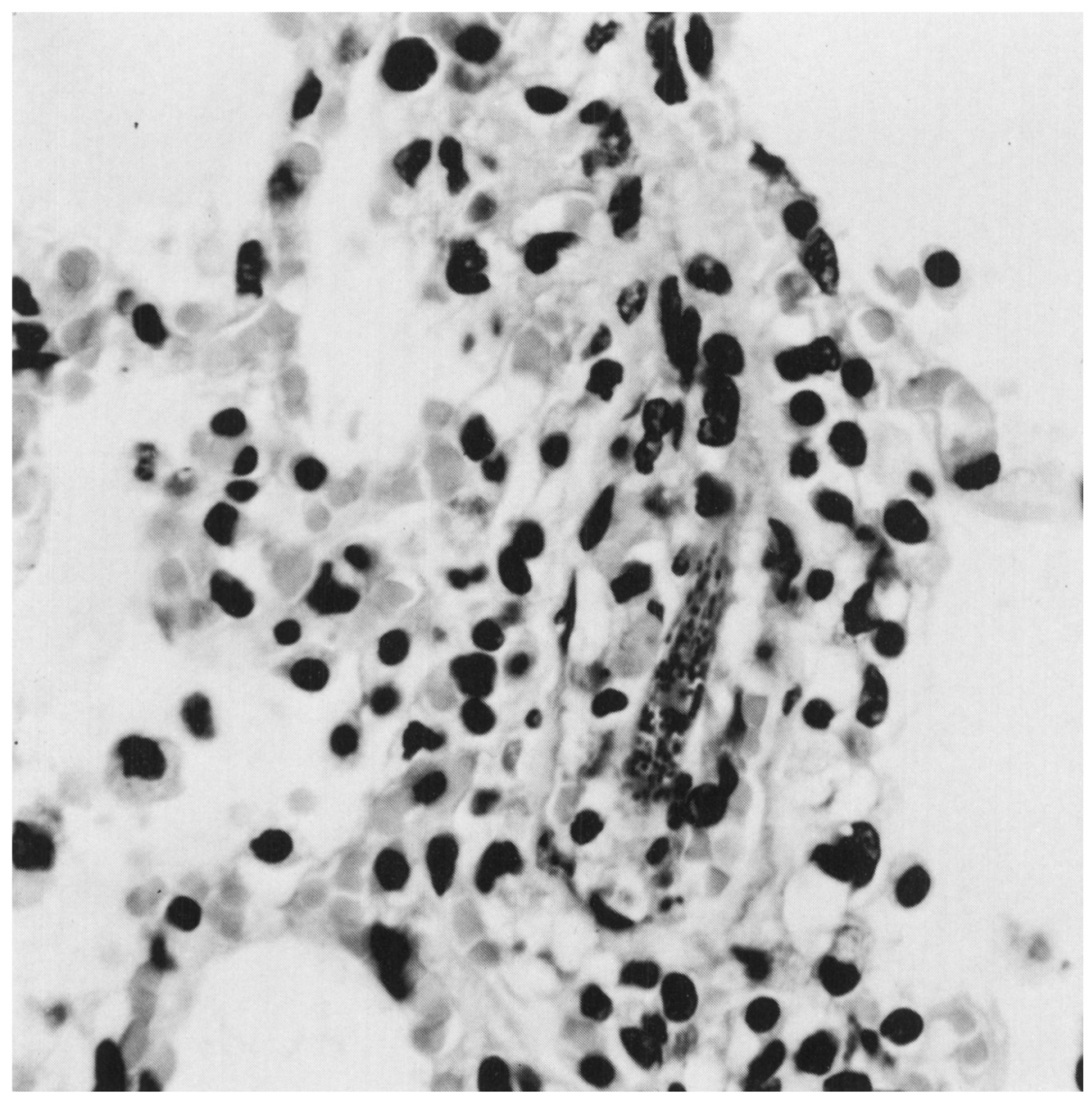

Figure 5-Portion of nematode lying within the lumen of a pulmonary capillary. H. and E. $x 800$.

similar to that of the genus Micronema (Goodey and Goodey, 1963).

Following receipt of Dr. Connor's report, unsuccessful attempts were made to macerate formalin-fixed brain tissue with pepsin and isolate whole free organisms. Aliquots of the cobalt formalin in which the brain had been fixed and preserved were then centrifuged, and free organisms in various stages of development as illustrated in Figures 7 , 8,9 and 10 were apparent. The largest nematode in these preparations measured 338 microns in length and contained an egg 35 microns in length.

Tissue and preparations of the centrifuged formalin were then submitted to the Ontario Veterinary College Department of Pathology, and the taxonomist confirmed the identification of the organism as Micronema deletrix.
This is a free living saprophagous nematode found naturally in decaying humus (Anderson and Bemrick, 1965). It is, however, known to be a facultative parasite, for it has been reported as the cause of a large granuloma in the maxilla and maxillary sinus of a horse (Johnson and Johnson, 1966). The organism was present in this equine granuloma in inordinate numbers, estimated at 87,500 per gram of tissue.

The pathogenesis of this verminous meningo-encephalomyelitis is of course speculative. It is assumed that nematodes were deposited in this child's lacerations at the time of the accident, and that some migrated to the central nervous system, multiplying in these tissues and inducing the inflammatory reaction noted histologically. This facultative parasite, like some other nematode species affecting animals and man may ex- hibit a preferential development in the central nervous system. There can be little doubt that they multiplied within the child's central nervous system, for they were widely disseminated, present in large numbers, and evident in all stages of development 24 days following the accident.

The cause of this child's meningo-encephalomyelitis was not anticipated at the time of autopsy, and his well-healed wounds, which exhibited no gross evidence of inflammation, were not examined histologically. Although a continued proliferation of organisms within a nidus in a healed wound cannot be excluded, it appears that the few juvenile forms of nematodes found within pulmonary capillaries 24 days after the accident were carried to that site from the central nervous system, rather than the reverse. The 


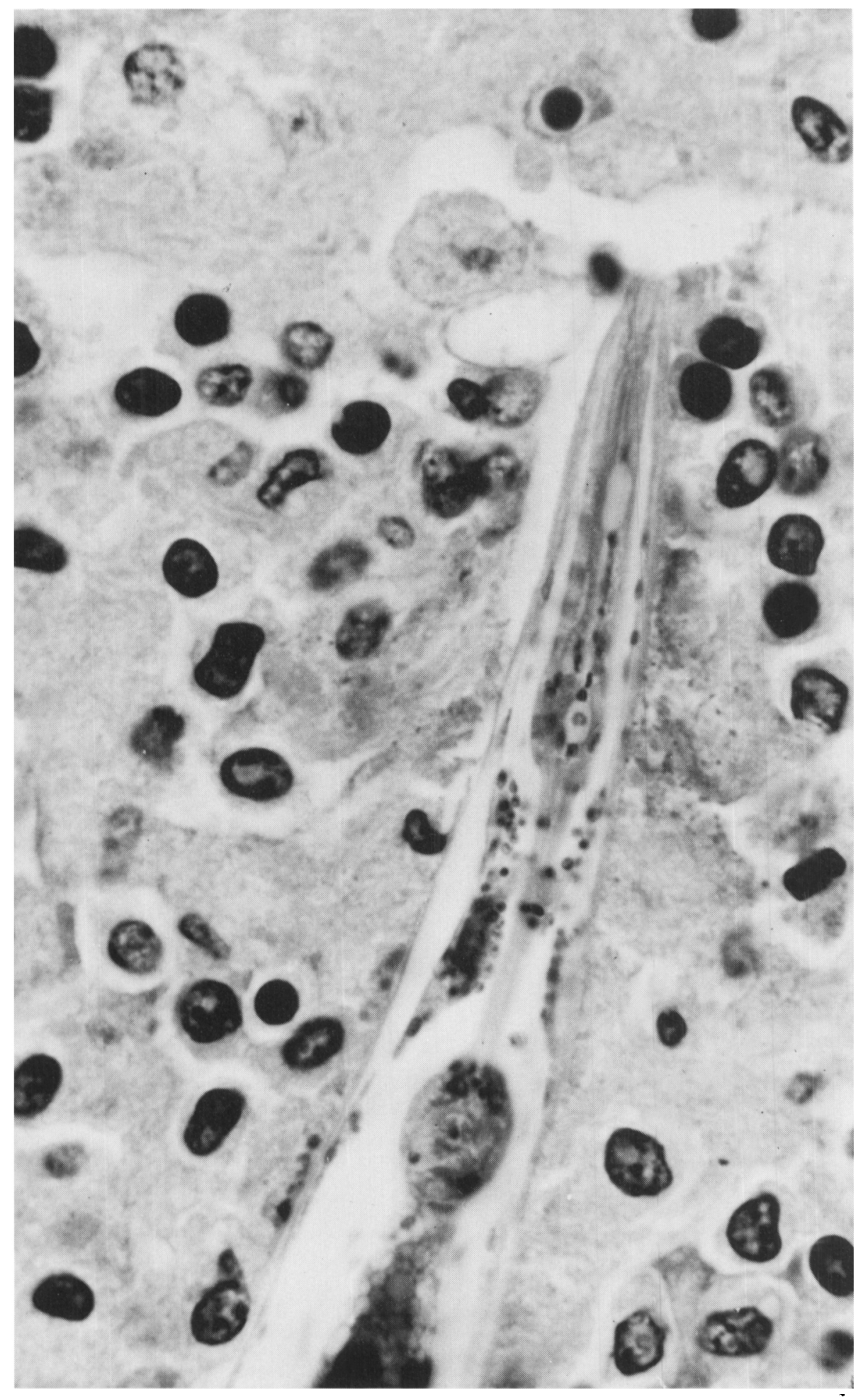

Figure 6-Foreparts of nematode. H. and E. x 1536.

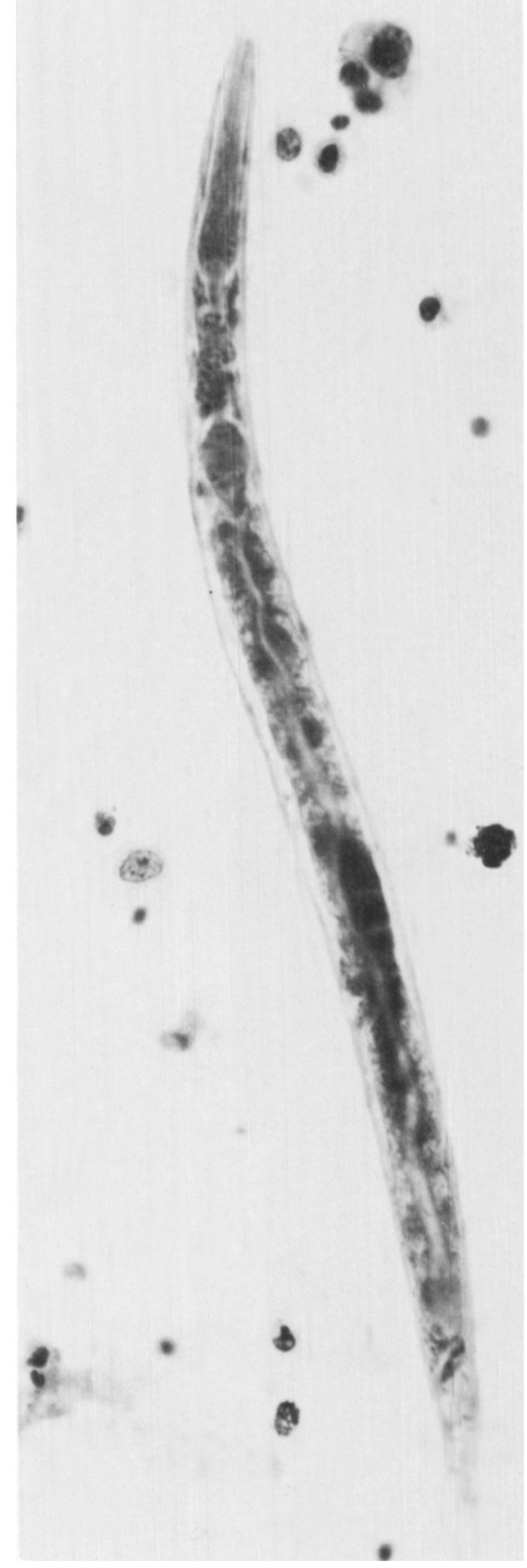

Figure 7-Nematode recovered from centrifuged formalin in which the brain had been suspended. It measures 290 microns in length and up to 15.6 microns in diameter. The total length of the oesophagus from the anterior extremity of the stoma to the cardia is 85 microns. The vulva is situated 180 microns from the anterior end, and the length of the tail, measured from anus to tip is 52 microns. H. and E. $\times 610$. 


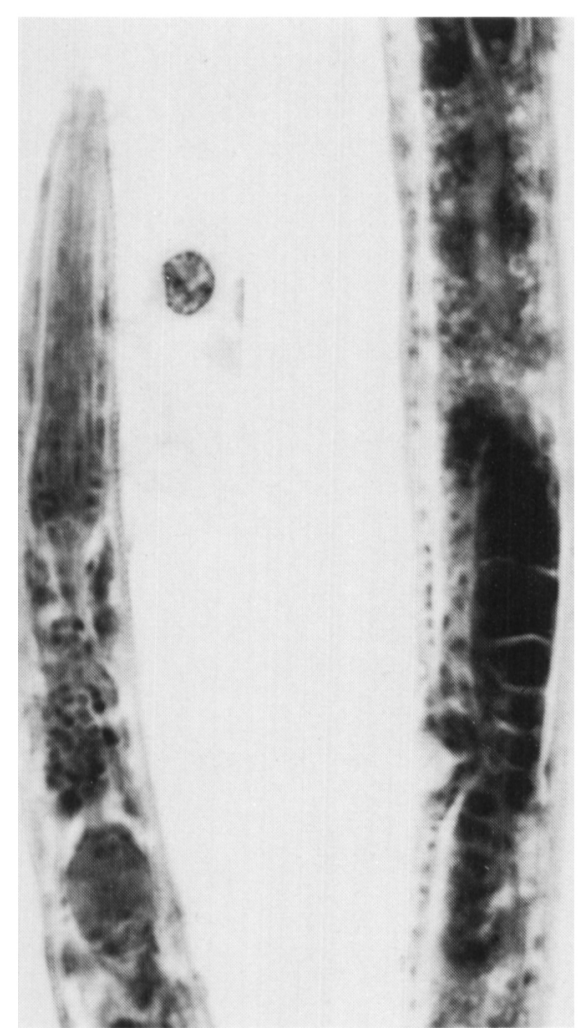

Figure 8-Foreparts and gonad of the nematode illustrated in Figure 8 . The fine annulations of the cuticle are just evident. The ovary extends caudal to the vulva and is reflexed dorsally. $\mathrm{H}$. and E. $x 1600$.

lack of hepatic involvement would appear to exclude intestinal infestation.

It would of course be desirable to identify Micronema in manure from the farm and site of the accident.

Samples of manure and soil were collected from this farm in the early spring of the year, 18 months following the accident. These samples were submitted to the Manitoba Provincial Veterinary Laboratory, but no nematodes were found.

The farm was visited again in October, three years following the accident. The original manure had been utilized during this interval, but samples of manure-soil mixture were again collected. Unlike the first samples which were obtained in the early spring while frost was in the ground, these samples now abounded in nematodes of various sizes and descriptions. While some were of comparable size to Mic-

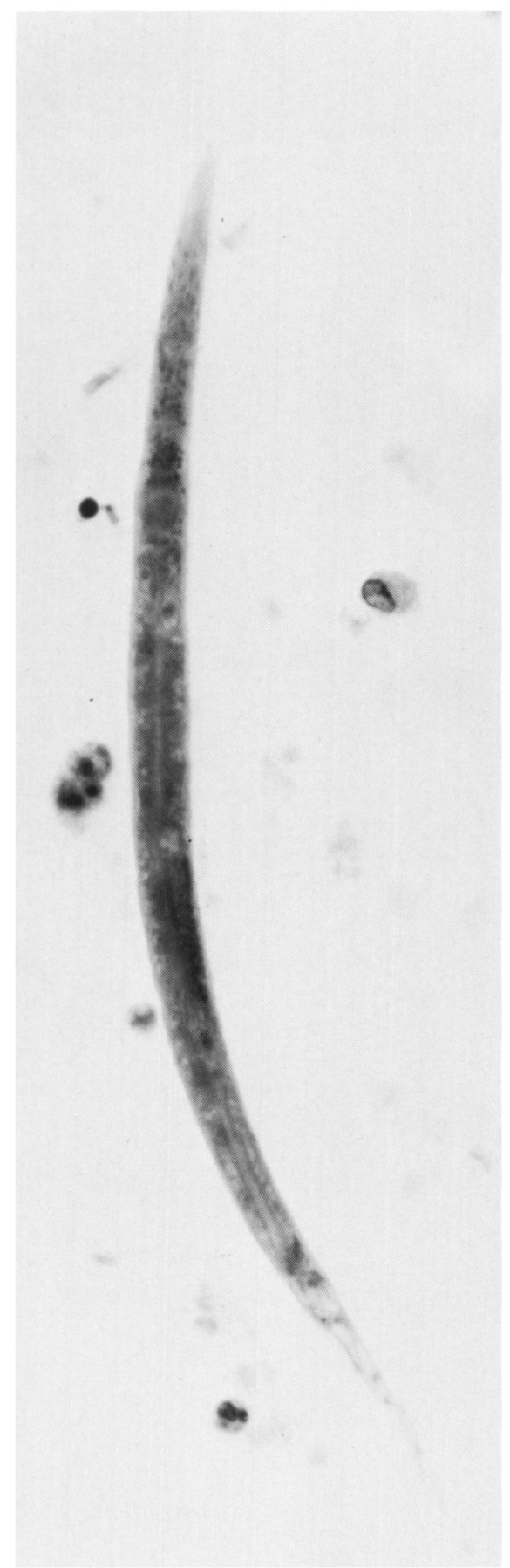

Figure 9-Juvenile or incompletely developed nematode. $H$. and $E$. x640.

ronema, none were identified as identical to that organism. The chances of recovering Micronema from the farm soil samples may have been influenced by the long interval between the child's accident and the subsequent searches for the offending organism.

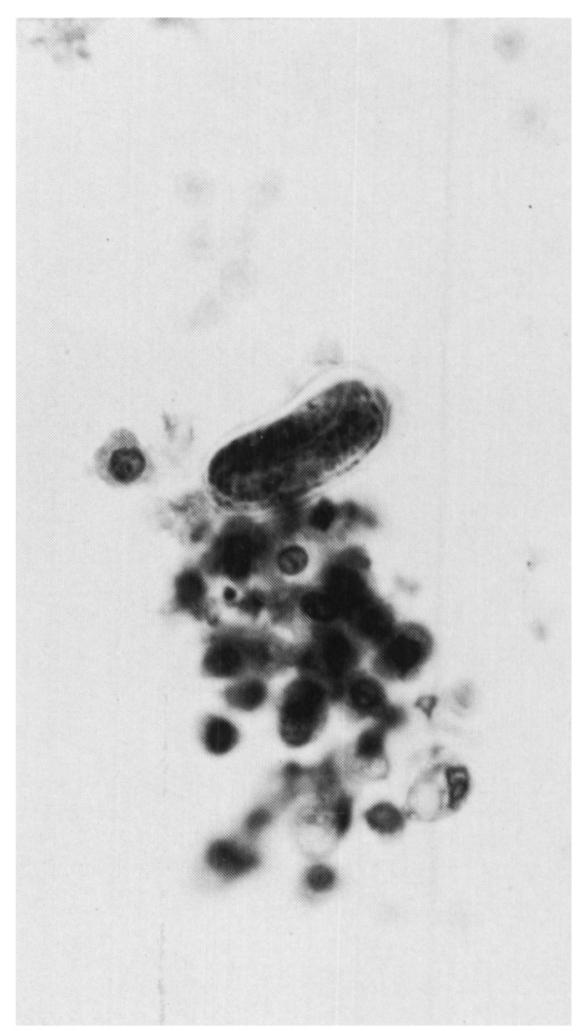

Figure 10-Early embryo lying folded within its covering. $H$. and $E$. x640.

While the pathogenic potentialities of this saprophagous nematode have now been demonstrated in man and the horse, it would be advisable to study these properties more thoroughly in experimental animals.

Hopefully, the verminous complication of this unusual farm accident is so rare, that it will never again be experienced.

\section{REFERENCES}

ANDERSON, R. V. and BEMRICK, W. J. (1965). Micronema deletrix n.sp., a saprophagous nematode inhabiting a nasal tumor of a horse. Proceedings of the Helminthological Society of Washington, 32: 74-75.

GOODEY, T. and GOODEY, J. B. (1963). Soil and freshwater nematodes, p. 263 Methuen and Co. Ltd., London.

JOHNSON, K. H. and JOHNSON, D. W. (1966). Granulomas associated with Micronema deletrix in the maxillae of a horse. Journal of the American Veterinary Medical Association 149: 155-159. 